\title{
Representation of 3D and 4D Objects Based on an Associated Curved Space and a General Coordinate Transformation Invariant Description
}

\author{
Eric Paquet \\ Visual Information Technology Group, National Research Council, M-50 Montreal Road, Ottawa, ON, Canada K1A 0R6
}

Received 25 January 2006; Revised 24 July 2006; Accepted 26 August 2006

Recommended by Petros Daras

This paper presents a new theoretical approach for the description of multidimensional objects for which 3D and 4D are particular cases. The approach is based on a curved space which is associated to each object. This curved space is characterised by Riemannian tensors from which invariant quantities are defined. A descriptor or index is constructed from those invariants for which statistical and abstract graph representations are associated. The obtained representations are invariant under general coordinate transformations. The statistical representation allows a compact description of the object while the abstract graph allows describing the relations in between the parts as well as the structure.

Copyright (C) 2007 Eric Paquet. This is an open access article distributed under the Creative Commons Attribution License, which permits unrestricted use, distribution, and reproduction in any medium, provided the original work is properly cited.

\section{INTRODUCTION}

Content-based description plays a prominent role in indexing and retrieval [1-4]. It is therefore important to develop invariant representations for 3D objects. An excellent review about indexing and retrieval of 3D objects can be found in [1-3]. As can be seen from this review, most of the proposed techniques are invariant under a very limited class of transformations, for example, translations, scaling, and rotations. Relatively less attention has been devoted to the development of representations that are invariant under general coordinate transformations. In addition, most approaches are limited to $3 \mathrm{D}$ objects understood in the sense of 2-D surfaces embedded in 3D space (e.g., a VRML object) and cannot be applied to volumetric objects, like those generated by tomography. Such multidimensional objects are characterised by the fact that each point in 3D space (volumetric space) is associated with a set of attributes. For instance, in the case of tomography, the set is generally limited to one attribute, the density, and the map is called a $4 \mathrm{D}$ object. This paper presents a new approach for invariant description of multidimensional objects under general coordinate transformations leading to a new type of representation based on the Ricci tensor and scalar. This novel approach transposes certain results of general relativity [5-7] and Riemannian geometry [5] into the framework of computer vision.
The paper is organised as follows. After some considerations on content-based indexing and retrieval, we review the most important results of tensor analysis which are necessary to understand our approach. Then, a tensor is associated to each object and the fundamental equations are derived from a variational principle. This tensor describes the attributes of the object and becomes the source for an associated curved space. The geometry of this associated curved space is described by a quadric form or a metric, a Ricci tensor and a Ricci scalar from which invariant quantities are derived. Finally, two representations are adopted for the invariants. The first one is a statistical representation based on a novel histogram and the second representation is a topological one based on an abstract graph. These representations are invariant under general coordinate transformations.

\section{CONTENT-BASED DESCRIPTION OF 3D AND 4D OBJECTS}

An important challenge in content-based description is to find a representation which is invariant under arbitrary coordinate transformations. Furthermore, such a description should not be limited to 3D objects, but should be easily extendable to multidimensional object (such as $4 \mathrm{D}$ tomography, as illustrated in Figure 1). The extension to 


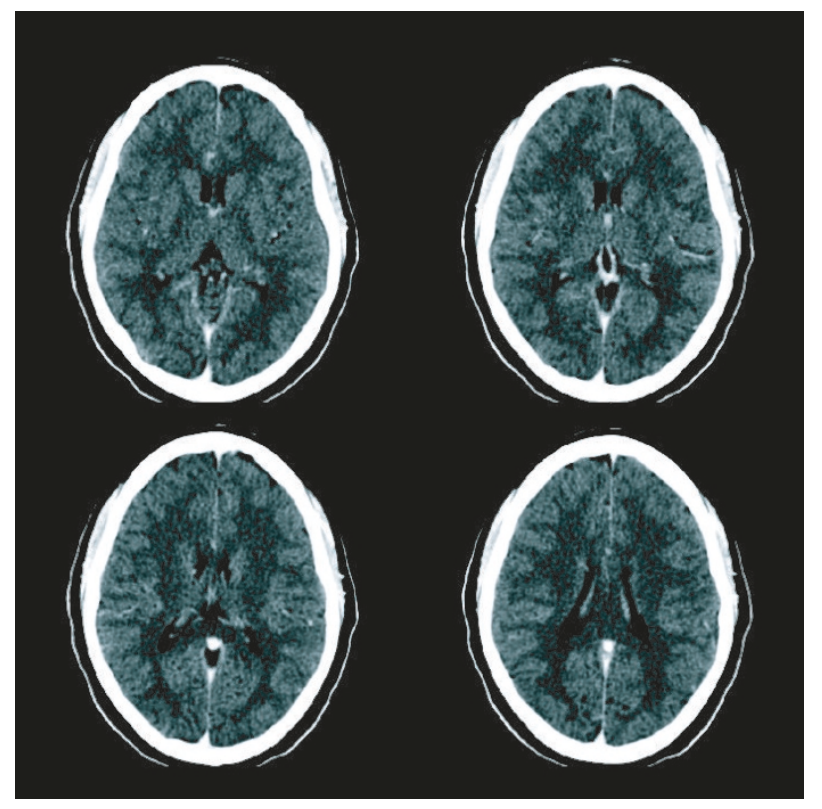

FIGURE 1: Four views of a tomographic image of the brain. The intensity is related to the density. Each slice corresponds to a certain elevation in the brain.

multidimensional content is problematic due to the high number of dimensions involved, their heterogeneity (space, time, speed, density, field intensity, etc.), and the fact that the standard mathematical framework has proven itself to be not suitable to derive form invariant equations under arbitrary coordinate transformations [5-7]. Form invariance is important in order to construct a description that is invariant under arbitrary coordinate transformations. This means that, no matter how the initial object is transformed (as defined in Section 3), the associated description is always the same. A new approach is presented to this paper, based on tensor analysis, in which a Riemannian space or curved space (as opposed to standard flat Euclidian space) is associated to an object. This space is described by tensorial equations which are form invariant under arbitrary coordinate transformations. A set of invariant quantities are extracted from this space and a representation is constructed from the set of invariants. Two types of representation are considered: Riemannian histograms and abstract graphs.

\section{AN OVERVIEW OF TENSOR ANALYSIS}

In this section, an overview of tensor algebra is presented. Derivations and more details can be found in [5-8]. This section is a prerequisite for what follows. Unless stated otherwise, all Greek indices and all summations are to be taken from 1 to $N$. Furthermore, if an index is not involved in a summation, it is immaterial and can be replaced by any other index.

The use of tensorial analysis is justified by the fact that tensorial equations do not change their form under arbitrary coordinate transformations. We assume that the space has an arbitrary number of dimensions, which means that the equations that we will derive could be applied to both $3 \mathrm{D}$ and $4 \mathrm{D}$ objects. A point in space is given by

$$
x=\left\{x^{\mu}\right\}
$$

For instance, in 3D

$$
x=\left(\begin{array}{l}
x_{1} \\
x_{2} \\
x_{3}
\end{array}\right)=\left(\begin{array}{l}
x \\
y \\
z
\end{array}\right)
$$

We assume that it is possible to associate to the space a metric $g_{\mu \nu}(x)$, which is defined by the quadratic form

$$
d s^{2} \equiv \sum_{\mu \nu} g_{\mu \nu}(x) d x^{\mu} d x^{\nu}
$$

In other words, we assume that we can define an invariant distance locally. This is an important distinction in between standard Euclidian geometry and Riemannian geometry: distance is a global invariant (for orthogonal transformations) for the former and a local invariant for the latter (under general coordinate transformations). Indeed, because of the space curvature, it is not possible to define a global metric. It should be noticed that $d s$, the infinitesimal length of arc, is an invariant and has the same value irrespectively of the coordinate system. Note that this metric defines the inner product between two tensors for the curved space.

The covariant and a contravariant vector are defined, respectively, as

$$
A_{\mu}^{\prime}\left(x^{\prime}\right)=\sum_{\nu} \frac{\partial x^{\nu}\left(x^{\prime}\right)}{\partial x^{\prime \mu}} A_{\nu}(x), \quad B^{\prime \mu}\left(x^{\prime}\right)=\sum_{\nu} \frac{\partial x^{\prime \mu}(x)}{\partial x^{\nu}} B^{\nu}(x),
$$

where

$$
x^{\prime \mu}=x^{\prime \mu}(x), \quad x^{\nu}=x^{\nu}\left(x^{\prime}\right)
$$

are 1 the general coordinate transformations or GCT. One should notice that such a transformation is local and completely general, except for the fact that it has to be continuously differentiable. That means that the GCT must be continuous and should not present any discontinuity at any order of derivation. The GCT can fluctuate rapidly but all derivatives must necessarily remain continuous. That means, for instance, that discrete coordinate transformations (reflections) are not allowed. As we know, the covariant and contravariant components associated with a vector in an orthogonal Euclidian reference frame are identical. If the Euclidian reference frame is not orthogonal, the contravariant and the covariant components are defined as the projections of the vector normal and parallel to the reference axes, respectively. 
Of course, if the axes of the reference frame are normal, the parallel and the normal projections are identical.

In general, $p$ contravariant and $q$ covariant tensors are defined as

$$
\begin{aligned}
& T^{{ }^{\prime}}{ }_{v^{\prime}{ }_{1} \cdots v^{\prime}{ }^{\prime}}^{\mu_{1} \cdots \mu^{\prime}{ }_{p}}\left(x^{\prime}\right) \\
& =\sum_{\mu_{1} \cdots \mu_{p}, v_{1} \cdots v_{q}} \frac{\partial x^{\prime \mu_{1}}(x)}{\partial x^{\mu_{1}}} \cdots \\
& \times \frac{\partial x^{\prime \mu_{p}}(x)}{\partial x^{\mu_{p}}} \frac{\partial x^{\nu_{1}}\left(x^{\prime}\right)}{\partial x^{\prime \nu_{1}^{\prime}}} \cdots \frac{\partial x^{\nu_{q}}\left(x^{\prime}\right)}{\partial x^{\prime \nu_{q}^{\prime}}} T_{\nu_{1}^{\prime} \cdots \nu_{q}}^{\mu_{1} \cdots \mu_{p}}(x) \text {. }
\end{aligned}
$$

At this point we should make a few remarks about the general coordinate transformations. It can be shown [5-8] that tensorial calculus is valid if and only if the general coordinate transformations are continuously differentiable which means that the transformations are continuous and smooth at any order. Furthermore, the mapping between the original coordinates and the transformed coordinates should be biunivoque which means that to each point corresponds one and only one transformed point, and vice versa. These constraints ensure that not only the tensorial equations are valid, but that an object cannot be transformed arbitrarily into another object. This is a fundamental requirement for searching and retrieval. Any transformation that satisfied the abovementioned requirements is compatible with our approach.

We will admit the following results without demonstration [5-8]. The metric is a symmetric tensor $g_{\mu \nu}(x)=g_{\nu \mu}(x)$. If a tensor is identically zero in a coordinate system, it is equal to zero in any other coordinate system. The product of a tensor by a tensor is a tensor and so is the sum. The symmetry and antisymmetry properties of a tensor are conserved under general coordinate transformations and so are the symmetry properties of the corresponding object. In addition, the most important property can be stated as follow: a tensorial equation does not change form under general coordinate transformations. Such a feature is highly desirable if one seeks to define quantities that are coordinate transformations invariant, that is, quantities that can describe an object irrespectively of its state of transformation. Furthermore, the following relations will be of use:

$$
\begin{gathered}
\sum_{\rho} \frac{\partial x^{\prime \mu}(x)}{\partial x^{\rho}} \frac{\partial x^{\rho}\left(x^{\prime}\right)}{\partial x^{\prime \nu}}=\delta_{\nu}^{\mu} \\
g_{\mu \nu}(x)=\sum_{\rho} \frac{\partial x^{\prime} \rho(x)}{\partial x^{\mu}} \frac{\partial x^{\prime}(x)}{\partial x^{\nu}} \\
\sum_{\rho} g^{\mu \rho}(x) g_{\nu \rho}(x)=\delta_{\nu}^{\mu} .
\end{gathered}
$$

The metric has an additional important property; it can transform covariant indices into contravariant indices and vice versa as illustrated by the following equation:

$$
A_{\mu \nu}(x)=\sum_{\alpha \beta} g_{\mu \alpha}(x) g_{\nu \beta}(x) A^{\alpha \beta}(x)
$$

The derivative of a tensor is not a tensor. Indeed, if one calculates the derivative of a covariant vector, one obtains

$$
\frac{\partial A_{\mu}^{\prime}\left(x^{\prime}\right)}{\partial x^{\prime \nu}}=\sum_{\rho \sigma} \frac{\partial x^{\rho}\left(x^{\prime}\right)}{\partial x^{\prime \mu}} \frac{\partial x^{\sigma}\left(x^{\prime}\right)}{\partial x^{\prime \nu}} \frac{\partial A_{\rho}\left(x^{\prime}\right)}{\partial x^{\prime \sigma}}+\sum_{\rho} A_{\rho}\left(x^{\prime}\right) \frac{\partial^{2} x^{\rho}\left(x^{\prime}\right)}{\partial x^{\prime \mu} \partial x^{\prime \nu}}
$$

The first term of the right member has the correct form as defined by (4), that is, it is transformed as a tensor, but the second term is incompatible with the definition of a tensor (the transformation involves the second-order derivative of $\left.x^{\rho}\left(x^{\prime}\right)\right)$. Nevertheless, one can define a covariant derivative, which is form invariant under general coordinate transformations

$$
\nabla_{\nu} A_{\mu}(x) \equiv \frac{\partial A_{\mu}(x)}{\partial x^{\nu}}-\sum_{\sigma} \Gamma_{\mu \nu}^{\sigma}(x) A_{\sigma}(x)
$$

where $\Gamma_{\mu \nu}^{\sigma}$ is named the affine connection and is related to the metric by the following relation:

$$
\Gamma_{\mu \nu}^{\sigma}(x)=\sum_{\rho} \frac{1}{2} g^{\sigma \rho}(x)\left(\frac{\partial g_{\mu \rho}(x)}{\partial x^{\nu}}+\frac{\partial g_{\rho \nu}(x)}{\partial x^{\mu}}-\frac{\partial g_{\mu \nu}(x)}{\partial x^{\rho}}\right) .
$$

It should be noticed that the affine connection is not a tensor. Irrespectively on the mathematics involved, the covariant derivative is a simple concept. The derivative in Euclidian space is related to the concept of slope or in other words the difference in between two points at two distinct positions. Such an operation is not problematical in standard Euclidian geometry since the space is flat, homogeneous, and isotropic. In our case, the space is not flat but curved and one cannot compare two points at two different locations because they live so to say in two different spaces. What can be done though is to transport one of the vector "parallel" to itself to the other location and then to compare them on the same location. This is what is expressed by (12) and the affine connection is responsible for such a parallel transportation.

Riemannian spaces are not conservative: if a vector is moved along a close path, the resulting vector does not coincide in general with the original vector. That means again that it is not possible to compare two tensors at two distinct positions. One can easily convince oneself of that. For example, it suffices to move a vector from the North Pole to the equator along a meridian, then once more along the equator, and finally back to the North Pole along a meridian: the initial and the final directions of the vector are different although the norm remains the same. Such a phenomenon happens because the Earth surface is a 2D curved surface: a sphere.

It becomes interesting then to characterise such behaviour by analysing the variation to which a vector is submitted if it is transported along an infinitesimal loop. For an infinitesimal closed path $C$, one can demonstrate that the 
variation is proportional to the curvature tensor

$$
\begin{aligned}
\Delta A_{\mu}(x) & =-\oint_{C} \sum_{\nu \sigma} \Gamma_{\mu \nu}^{\sigma}(x) A_{\sigma}(x) d x^{\nu} \\
& =\frac{1}{2} \sum_{\nu \sigma \tau} R_{\mu \sigma \tau}^{\nu}(x) A_{\nu}(x) d x^{\sigma} \wedge d x^{\tau},
\end{aligned}
$$

where $\wedge$ represents the external product and where the Riemannian curvature tensor $R_{\mu \sigma \tau}^{v}(x)$ is defined as

$$
\begin{aligned}
R_{\mu \sigma \tau}^{v}(x) \equiv & \frac{\partial \Gamma_{\mu \tau}^{\nu}(x)}{\partial x^{\sigma}}-\frac{\partial \Gamma_{\mu \sigma}^{\nu}(x)}{\partial x^{\tau}} \\
& +\sum_{\rho} \Gamma_{\rho \sigma}^{\nu}(x) \Gamma_{\mu \tau}^{\rho}(x)-\Gamma_{\rho \tau}^{\nu}(x) \Gamma_{\mu \sigma}^{\rho}(x) .
\end{aligned}
$$

From the inner product between the metric and the Riemannian curvature tensor, one can define the Ricci tensor $R_{v \sigma}(x)$ and the Ricci scalar $R(x)$ which are, respectively, given by

$$
\begin{gathered}
R_{\nu \sigma}(x)=\sum_{\mu \tau \rho} g^{\mu \rho}(x) g_{\mu \tau}(x) R_{\nu \rho \sigma}^{\tau}(x) \\
R(x)=\sum_{\nu \sigma} g^{\nu \sigma}(x) R_{\nu \sigma}(x)
\end{gathered}
$$

The Ricci tensor is symmetric. The Ricci tensor and scalar satisfy many identities among which are the Bianchi identities which are more or less a conservation identity:

$$
\sum_{\alpha} \nabla_{\alpha}\left(R^{\sigma \alpha}(x)-\frac{1}{2} g^{\sigma \alpha} R(x)\right)=0 .
$$

The Riemann tensor and the Ricci tensor and scalar characterise the curvature of the space at a given point. The Riemann and the Ricci tensor are not invariant under general coordinate transformations: they transform as tensors. However, the Ricci scalar is an invariant: its value is the same irrespectively of the general coordinate transformation applied. Such a feature is common to all scalars in a Riemannian space. At this stage, it is important to realise that the curvature is not bonded to a particular coordinate system but to the physical point itself. For instance, even if the Ricci scalar is an invariant, the coordinates of the point to which it is attached change under a GCT. In Section 5, we will see how we can represent invariant quantities independently of their coordinates.

There is a relation in between the Ricci scalar and the standard intrinsic Gaussian curvatures. One can demonstrate that in 2-D, the Ricci scalar and the intrinsic Gaussian curvatures are related by the relation

$$
{ }^{(2)} R(x) \propto \kappa_{1}(x) \kappa_{2}(x),
$$

where $\kappa_{1}(x)$ and $\kappa_{2}(x)$ are the intrinsic Gaussian curvatures. Such a relation does not exist in 3D or in higher dimensions. From that point of view, the Ricci scalar can be considered to a generalisation of the intrinsic curvatures to three dimensions and more.

\section{ASSOCIATION OF A CURVED SPACE WITH AN OBJECT}

In this section, a Riemannian curved space is associated with an object. A set of equations that are form invariant under general coordinate transformations are derived. In order to construct those equations, a tensor is associated with the object. Such an association can be realised, for instance, through the density of a tomographic image. This tensor acts as a source term in a field equation from which the geometry of the associated curved space is calculated.

The formulation of form invariant equations under general coordinate transformations is a complex task. It would be much easier if one could associate and define an invariant scalar functional from which the equations could be derived. Such an approach has been developed: the scalar functional is the Lagrangian and the equations are derived from a variational principle or least action principle. For our purpose, the Lagrangian is a scalar functional related to the "energy of the system." The energy could be related to the density of a tomographic image, the 3D shape deformation (like a deformable mesh), or some topological characteristics (like the number of holes in a certain neighbourhood). The reader who is not familiar with Lagrangians and variational principles is referred to $[5,8]$ for more details. Once the Lagrangian is defined, one can derive the corresponding equations by finding the extremum for the action associated with the Lagrangian. We formulate the Lagrangian in such a way that it incorporates all our requirements about the curved space we want to associate with the object as well as all our knowledge (from an indexing and retrieval point of view) about the object itself.

In a Riemannian space, the action $S$ [5-7] is defined as

$$
S \equiv \int d^{N} x \sqrt{-\operatorname{det}\left(g_{\mu \nu}(x)\right)} \mathcal{L}\left[g_{\mu \nu}(x)\right]
$$

where $\mathcal{L}$ is the Lagrangian (strictly speaking the Lagrangian density) and $\operatorname{det}\left(g_{\mu \nu}(x)\right)$ the determinant of the metric (the metric is a matrix). One should notice that the action is also a scalar and consequently invariant under a GCT. The extra factor $\sqrt{-\operatorname{det}\left(g_{\mu \nu}(x)\right)}$ is related to the Jacobian of the transformation and ensures that the result of the integration does not depend on a particular choice of the coordinate system; in other words, the infinitesimal volume element (or hypervolume) does not depend on the reference frame employed.

The principle of least action [5-8] states that if the action is extremal, the Lagrangian necessarily satisfies the EulerLagrange equations, which can be written in our specific case as

$$
\begin{gathered}
\sum_{\rho} \frac{\partial}{\partial x_{\rho}} \frac{\delta\left(\sqrt{-\operatorname{det}\left(g_{\mu \nu}(x)\right)} \mathcal{L}\left[g_{\mu \nu}(x)\right]\right)}{\delta\left(\partial g_{\mu \nu}(x) / \partial x_{\rho}\right)} \\
-\frac{\delta\left(\sqrt{-\operatorname{det}\left(g_{\mu \nu}(x)\right)} \mathcal{L}\left[g_{\mu \nu}(x)\right]\right)}{\delta g_{\mu \nu}(x)}=0,
\end{gathered}
$$


where $\delta h[f(x)] / \delta f(x)$ stands for the functional derivative (the derivative is calculated with respect to a function).

We are now in position to set our hypothesis and derive the corresponding equations. Let us assume that our Lagrangian can be split into two Lagrangians. The first Lagrangian $\hat{\mathscr{L}}\left[g_{\mu \nu}(x)\right]$ depends solely on the metric and characterises the Riemannian space per se (the space we want to associate with our object) while the second Lagrangian $\breve{\mathscr{L}}\left[g_{\mu \nu}(x), \Phi(x)\right]$ depends on the metric and some other tensors $\Phi$ that characterise the object under consideration (e.g., the density in a volumetric image). It is very important to understand this point, the space associated with an object is not static but dynamics and its configuration depends on the energy content of the associated object. An analogy, although imperfect, is the association of a magnetic field to a current. As the current is the source of the magnetic field, the object is the source of the associated curved Riemannian space.

With these hypotheses in mind, the action can be written as

$$
S=\int d^{N} x \sqrt{-\operatorname{det}\left(g_{\mu \nu}(x)\right)}\left(\hat{\mathscr{L}}\left[g_{\mu \nu}(x)\right]+\breve{\mathscr{L}}\left[g_{\mu \nu}(x), \Phi(x)\right]\right) .
$$

Let us write the Lagrangian for the associated curved space. We have seen earlier that a curved space can be characterised by a set of curvatures: the simplest one being the Ricci scalar. Consequently, one of the simplest Lagrangian that can be constructed from the Riemannian curvatures is the one constructed from the Ricci scalar:

$$
\widehat{\mathscr{L}}\left[g_{\mu \nu}(x)\right]=\kappa^{-1} R(x)=\kappa^{-1} \sum_{\mu \nu} g_{\mu \nu}(x) R^{\mu \nu}(x),
$$

where $\kappa$ is an arbitrary constant. Of course this is not the only possibility. One could take, for instance, the tensorial product of a covariant and contravariant Ricci tensor but that would lead to unnecessarily complicated equations. For our purpose, we will be satisfied with the simplest form possible. If one substitutes (23) into (22) and optimised the action with (21), one obtains

$$
\delta S=0 \Longrightarrow R_{\mu \nu}(x)-\frac{1}{2} g_{\mu \nu}(x) R(x)-\kappa \breve{T}_{\mu \nu}(x)=0,
$$

where $\breve{T}_{\mu v}$, the source tensor, is associated with the object and is defined as

$$
\breve{T}_{\mu \nu}(x) \equiv \frac{\delta\left(\sqrt{-\operatorname{det}\left(g_{\mu \nu}(x)\right)} \breve{L}\left[g_{\mu \nu}(x), \Phi(x)\right]\right)}{\delta g_{\mu \nu}(x)} .
$$

Because of (18) and (24), the source tensor satisfies the Bianchi identities and is symmetric. In other words, only those source tensors for which the covariant divergence is zero are acceptable. Consequently, when defining the source tensor, one has to be very careful in order to verify that the covariant divergence of (25) is effectively zero.

Next, one can demonstrate that the source tensor is related to the density, the momentum, and the flux of momentum. For instance, for a static volumetric image one can de- fine the source tensor as

$$
\breve{T}_{00}(x)=\rho(x)
$$

and zero otherwise, that is, the tensor is simply related to the density. In the general case, the source tensor is more complicated. More details can be found in [5-8] but the general approach is well known. One defines a Lagrangian that characterised the energy content of the object under consideration. Such a characterisation might be either physical (e.g., real physical density), topological, or formal. Then the source tensor is calculated from (25).

Finally, if one substitutes the value of the source tensor in (24), one obtains

$$
R_{\mu \nu}(x)-\frac{1}{2} g_{\mu \nu}(x) R(x)=\kappa \breve{T}_{\mu \nu}(x)
$$

which is a set of ten (because of the symmetry properties of the tensors) form invariant nonlinear equations describing the relations in between the source tensor associated with the object and the curvatures of the corresponding Riemannian space. That is the relations we were looking for; we have associated a curved space to the object.

In addition, it can be demonstrated [5-8] that the mapping in between the source tensor (i.e., the object) and the Riemannian space is unique and consequently not ambiguous. This result is valid as long as the general coordinate transformations and the source tensor are continuously differentiable. That does not mean that transformations cannot vary rapidly, it only means that there should be no discontinuities (in the mathematical senses) in the transformations.

The solution of (27) is a highly nontrivial task. Nevertheless, a numerical solution can be obtained by foliating the space; see, for instance, [9].

\section{DEFINITION OF INVARIANT REPRESENTATIONS FROM A STATISTICAL REPRESENTATION AND FROM AN ABSTRACT GRAPH}

Up to this point, we have associated a Riemann space to an object and we have characterised the curvature of this space by calculating the Ricci tensor and scalar distributions. Now, in order to obtain an invariant description, we must construct some invariant quantities from the Ricci curvatures. If one applies a coordinate transformation to the Ricci scalar, one obtains with the help of (7) and (17)

$$
R^{\prime}=\sum_{\mu \nu} g^{\prime \mu \nu} R_{\mu \nu}^{\prime}=R
$$

Equation (28) shows that the Ricci scalar is invariant under arbitrary coordinate transformations and as a result we define our first ensemble of invariant quantities $\mathfrak{R}_{1}(x)$ as the ensemble

$$
\left\{\mathfrak{R}_{1}(x) \mid \mathfrak{R}_{1}(x) \equiv R^{2}(x)\right\}
$$


If one computes the tensorial product of a covariant and a contravariant Ricci tensor, one obtains

$$
\begin{aligned}
\sum_{\mu \nu} R_{\mu \nu}^{\prime} R^{\prime \mu \nu} & =\sum_{\mu \nu \rho \sigma}\left(\frac{\partial x^{\mu}}{\partial x^{\prime} \rho} \frac{\partial x^{\nu}}{\partial x^{\prime \sigma}}\right) R_{\mu \nu}\left(\frac{\partial x^{\prime} \rho}{\partial x^{\mu}} \frac{\partial x^{\prime \sigma}}{\partial x^{\nu}}\right) R^{\mu \nu} \\
& =\sum_{\mu \nu} R_{\mu \nu} R^{\mu \nu}
\end{aligned}
$$

which is again invariant under arbitrary coordinate transformations. Consequently, we define our second ensemble of invariant quantities $\mathfrak{R}_{2}(x)$ as

$$
\left\{\mathfrak{R}_{2}(x) \mid \mathfrak{R}_{2}(x) \equiv \sum_{\mu \nu} R_{\mu \nu}(x) R^{\mu \nu}(x)\right\} .
$$

As a result, an invariant statistical representation of the object can be constructed. The ensembles defined by (29) and (31) are described by two histograms. The first histogram characterises the distribution of the Ricci scalars while the second histogram characterised the distribution of the inner products of the Ricci tensors. More precisely, the histograms are defined as

$$
h_{k}(i) \equiv \sum_{\left\{x \mid\left[\left(i \Delta_{k}-\Delta_{k} / 2\right) \leq \mathfrak{R}_{k}(x)<\left(i \Delta_{k}+\Delta_{k} / 2\right)\right]\right\}} \mathfrak{R}_{k}(x),
$$

where $\Delta_{k}$ is the width of each bin for histogram $k$. In other words, the histograms provide a statistical distribution for the invariants: they do not depend on the location of the invariants on the object but only on their statistical distribution. Such a distribution is invariant under a CGT and characterises the object.

For retrieval purpose, these histograms can be considered as feature vectors and compared with standard techniques such as those described in $[1,2]$. For instance, comparison can be performed with a metric (distance), a correlation technique, a neural network, or with a Bayesian approach. Besides, whatever the method employed is, it is important that a certain degree of cross-correlation (bins with different indexes) be present in the comparison algorithm because the invariants, as defined by (29) and (32), may possibly present a certain bin index tolerance due to noise and inadequate sampling which means that bins could be shifted and the corresponding histograms distorted. For the metric approach, such a requirement can be implemented with a quadratic form.

An abstract graph representation is also possible. For such a graph, each point for which invariants are calculated is mapped to a node. Each node is related to the pair of invariants calculated at the corresponding point and not to the coordinates of the points, which are in any case arbitrary. The only relations that are invariant, irrespectively of the GCT applied to the object, are the adjacency relations in between the points. Such topological relations remain always the same, because the general coordinate transformations are continuously differentiable by hypothesis. The graph is then constructed in such a way that adjacent nodes (i.e., points) are connected by lines or links. The link indicates only a connection in between two nodes; the length of the link has no meaning per se, since the representation has to be invariant under a GCT. Such a graph is invariant under a GCT. The abstract graph obtained can be compared to another graph using standard techniques $[1,2]$.

The histogram representation is much more compact and is adapted to very large databases. The compactness is obtained at the price of loosing the adjacency relations. The abstract graph approach preserves those relations, but the size of the graph limits its applicability to small subset of data for which a detailed representation might be needed.

\section{PRACTICAL CONSIDERATIONS}

The proposed method may be applied to a wide class of $3 \mathrm{D}$ objects. Nevertheless, there are some restrictions that should be taken into account; for instance, the objects under consideration should be Riemannian manifolds. In essence, a manifold is a surface (or a volume) that can be defined by a set of overlapping patches. The surface, included in the overlapping regions, should be continuously differentiable. Such a case is approximated, for instance, by the NURBS or nonuniform rational $\beta$-splines which are widely utilise in computer graphics. The approximation comes from the fact that, in the NURBS representation, the overlapping regions are differentiable only up to a certain order. In addition to be a manifold, the surface should be Riemannian. That means that the surface should not present any torsion or, in other words, should not be twisted. For instance, if one cuts a circular band, twists the two extremities, and assembles them back together, one obtains a surface with torsion which cannot be described by the present approach. Otherwise, there are no restrictions and the considered surface can present holes, missing polygons, or other types of degeneracy.

For the vast majority of cases of interest, (27) must be solved numerically. As pointed out in [9], this is a difficult task in the sense that (27) is a set of 10 nonlinear differential equations. It has been shown [9] that such a set of equations can be numerically unstable if the numerical algorithm is not carefully designed: for instance, some constrains, like the Bianchi identities, that is, (18), must be enforced throughout the calculation. That means that for any practical application the calculation of the invariant representation must be performed offline. On the other hand, the retrieval operation can be performed in real time since the later involves only the comparison of histograms or graphs for which many real-time comparison approaches exist [1].

At this point, we would like to provide an illustrative example in order to better understand the proposed approach. Let us assume that we have a $3 \mathrm{D}$ object for which we have calculated the invariants as defined by (28) to (32). We would like to understand better the meaning of a GCT and how it generalises the traditional approaches. For instance, most invariant representations for 3D objects are rotation invariant. That means that a unique invariant description can be obtained independently of the orientation of the object in space. Such invariance is global since the object is rotated as a rigid solid. With our approach, it is possible to generalise this invariance to local rotations. By a local rotation we mean 
that the associated rotation matrix is a function of the coordinates on the object. Let us consider a GCT as defined by (4). Such an equation may be expressed in a matrix form as follows:

$$
\left[\begin{array}{l}
A_{0}^{\prime}\left(x^{\prime}\right) \\
A_{2}^{\prime}\left(x^{\prime}\right) \\
A_{3}^{\prime}\left(x^{\prime}\right)
\end{array}\right]=\left[\begin{array}{ccc}
\frac{\partial x_{0}\left(x^{\prime}\right)}{\partial x_{0}^{\prime}} & \frac{\partial x_{1}\left(x^{\prime}\right)}{\partial x_{0}^{\prime}} & \frac{\partial x_{2}\left(x^{\prime}\right)}{\partial x_{0}^{\prime}} \\
\frac{\partial x_{0}\left(x^{\prime}\right)}{\partial x_{1}^{\prime}} & \frac{\partial x_{1}\left(x^{\prime}\right)}{\partial x_{1}^{\prime}} & \frac{\partial x_{2}\left(x^{\prime}\right)}{\partial x_{1}^{\prime}} \\
\frac{\partial x_{0}\left(x^{\prime}\right)}{\partial x_{2}^{\prime}} & \frac{\partial x_{1}\left(x^{\prime}\right)}{\partial x_{2}^{\prime}} & \frac{\partial x_{2}\left(x^{\prime}\right)}{\partial x_{2}^{\prime}}
\end{array}\right]\left[\begin{array}{l}
A_{0}(x) \\
A_{1}(x) \\
A_{2}(x)
\end{array}\right]
$$

The transformation matrix, which in fact is a matrix functional, is invertible by construction since the matrix elements are continuously differentiable. This transformation is extremely general in the sense that the invariant representation does not depend on the form of this matrix. As a matter of fact, this matrix belongs to the group (in the mathematical sense) GL(3) of invertible matrices. We can consider a subgroup of GL(3): for instance, all the matrices for which the inverse is equal to the transpose of the transformation matrix. Such a matrix is the rotation matrix, that is, the group $O(3)$ of orthogonal matrices. Consequently, we have demonstrated that our approach is not only invariant for local rotations but also for much more general transformations. Consequently, our approach is a generalisation of global rotation invariance to local rotation invariance; in other words to local deformations.

\section{EXPERIMENTAL RESULTS}

In this section, we present experimental results. Our objective is to better understand invariants (29) and (31). Firstly, we prove that they are invariant under a general coordinate transformation by explicitly applying such a transformation. Then, we evaluate invariant (29) for particular symmetries of the source tensor. We do not present any evaluation of invariants (31) because they are too cumbersome. These calculations are performed for both $3 \mathrm{D}$ and $4 \mathrm{D}$ objects, in order to better understand the differences in between the two. All the results that follow have been obtained symbolically with the Wolfram Research Mathematica software. All the calculations were performed without any approximation. Consequently, the obtained results are exact. They can be utilised either as analytical expressions or as formulas in numerical evaluations. The results are presented with the Mathematica notation [10].

Firstly, we want to prove that our invariants are indeed invariant under a general coordinate transformation or GCT. For this purpose, we apply a GCT to invariant (29) and (31). The calculation is completely general and is performed for both $3 \mathrm{D}$ and $4 \mathrm{D}$ objects.

First, let us consider the case of three-dimensional objects. If we make the summation explicit, invariant (29) could be written as

$$
\left(R_{11}\right)\left(R^{11}\right)+2\left(R_{21}\right)\left(R^{12}\right)+\left(R_{22}\right)\left(R^{22}\right) .
$$

If we apply a GCT $\left(x^{\prime}=f 1[x, y], y^{\prime}=f 2[x, y]\right)$ to this invariant, we obtain

$$
\begin{aligned}
& \left(\left(\left(R^{22}\right) f 1^{(0,1)}[x, y]^{2}+2\left(R^{12}\right) f 1^{(0,1)}[x, y] f 1^{(1,0)}[x, y]\right.\right. \\
& \left.+\left(R^{11}\right) f 1^{(1,0)}[x, y]^{2}\right)\left(\left(R_{11}\right) f 2^{(0,1)}[x, y]^{2}\right. \\
& \left.\left.\quad-2\left(R_{21}\right) f 2^{(0,1)}[x, y] f 2^{(1,0)}[x, y]+\left(R_{22}\right) f 2^{(1,0)}[x, y]^{2}\right)\right) \\
& /\left(f 2^{(0,1)}[x, y] f 1^{(1,0)}[x, y]-f 1^{(0,1)}[x, y] f 2^{(1,0)}[x, y]\right)^{2} \\
& +\left(\left(\left(R_{11}\right) f 1^{(0,1)}[x, y]^{2}-2\left(R_{21}\right) f 1^{(0,1)}[x, y] f 1^{(1,0)}[x, y]\right.\right. \\
& \left.+\left(R_{22}\right) f 1^{(1,0)}[x, y]^{2}\right)\left(\left(R^{22}\right) f 2^{(0,1)}[x, y]^{2}\right. \\
& \left.\left.+2\left(R^{12}\right) f 2^{(0,1)}[x, y] f 2^{(1,0)}[x, y]+\left(R^{11}\right) f 2^{(1,0)}[x, y]^{2}\right)\right) \\
& /\left(f 2^{(0,1)}[x, y] f 1^{(1,0)}[x, y]-f 1^{(0,1)}[x, y] f 2^{(1,0)}[x, y]\right)^{2} \\
& +\left(2 \left(f 1^{(1,0)}[x, y]\left(\left(R_{21}\right) f 2^{(0,1)}[x, y]-\left(R_{22}\right) f 2^{(1,0)}[x, y]\right)\right.\right. \\
& \left.+f 1^{(0,1)}[x, y]\left(-\left(R_{11}\right) f 2^{(0,1)}[x, y]+\left(R_{21}\right) f 2^{(1,0)}[x, y]\right)\right) \\
& \quad \times\left(f 1^{(1,0)}[x, y]\left(\left(R^{12}\right) f 2^{(0,1)}[x, y]+\left(R^{11}\right) f 2^{(1,0)}[x, y]\right)\right. \\
& \left.\left.+f 1^{(0,1)}[x, y]\left(\left(R^{22}\right) f 2^{(0,1)}[x, y]+\left(R^{12}\right) f 2^{(1,0)}[x, y]\right)\right)\right) \\
& /\left(f 2^{(0,1)}[x, y] f 1^{(1,0)}[x, y]-f 1^{(0,1)}[x, y] f 2^{(1,0)}[x, y]\right)^{2}, \\
& +
\end{aligned}
$$

where $(1,0)$ indicates a partial derivative with respect to $y$ and $x$. A similar notation applies to other derivatives. Expression (35) reduces, after simplification, to expression (34) which proves the invariance of (29). One should notice that we need only two coordinates for a three-dimensional object since the later is a surface in three dimensions that can be parameterised with two and only two parameters.

Let us consider the case of $4 \mathrm{D}$ objects. If we make the summation explicit, invariant (29) can be written as

$$
\begin{aligned}
& \left(R_{11}\right)\left(R^{11}\right)+2\left(R_{21}\right)\left(R^{12}\right)+2\left(R_{31}\right)\left(R^{13}\right) \\
& \quad+\left(R_{22}\right)\left(R^{22}\right)+2\left(R_{32}\right)\left(R^{23}\right)+\left(R_{33}\right)\left(R^{33}\right) .
\end{aligned}
$$

If we apply a GCT $\left(x^{\prime}=f 1[x, y, z], y^{\prime}=f 2[x, y, z], z^{\prime}=\right.$ $f 3[x, y, z])$ to this invariant, we obtain a lengthy expression (10 pages), which simplifies to (36) after a tedious calculation. Once more, we need three coordinates because a $4 \mathrm{D}$ object is a volume that can be parameterised with three and only three coordinates.

We now calculate invariant (29) for some particular cases. It is possible to perform an exact calculation for the invariant if some kind of symmetry is assumed for the source tensor and consequently for the metric. We consider both 3D and $4 \mathrm{D}$ objects.

We first address the case of 3D objects. In the particular case of a three-dimensional object, invariant (29) can be calculated for a general metric. In that case, only two coordinates are needed since a $3 \mathrm{D}$ object is a surface that can be parameterised with two coordinates. If we perform the 
calculations, we obtain

$$
\begin{aligned}
(g 11[ & x, y]\left(g 11^{(0,1)}[x, y] g 22^{(0,1)}[x, y]-2 g 22^{(0,1)}[x, y]\right. \\
& \left.\times g 21^{(1,0)}[x, y]+g 22^{(1,0)}[x, y]^{2}\right)+g 21[x, y] \\
& \times\left(g 22^{(0,1)}[x, y] g 11^{(1,0)}[x, y]+2 g 21^{(1,0)}[x, y]\right. \\
& \times\left(2 g 21^{(0,1)}[x, y]-g 22^{(1,0)}[x, y]\right)-g 11^{(0,1)}[x, y] \\
& \left.\times\left(2 g 21^{(0,1)}[x, y]+g 22^{(1,0)}[x, y]\right)\right)+2 g 21[x, y]^{2} \\
& \times\left(g 11^{(0,2)}[x, y]-2 g 21^{(1,1)}[x, y]+g 22^{(2,0)}[x, y]\right) \\
& +g 22[x, y]\left(g 11^{(0,1)}[x, y]^{2}+g 11^{(1,0)}[x, y]\right. \\
& \times\left(-2 g 21^{(0,1)}[x, y]+g 22^{(1,0)}[x, y]\right)-2 g 11[x, y] \\
& \left.\left.\times\left(g 11^{(0,2)}[x, y]-2 g 21^{(1,1)}[x, y]+g 22^{(2,0)}[x, y]\right)\right)\right)^{2} \\
/( & \left.4\left(g 21[x, y]^{2}-g 11[x, y] g 22[x, y]\right)^{4}\right) .
\end{aligned}
$$

Equation (37) reduces to

$$
\begin{aligned}
& \left(g 11[x, y]\left(g 11^{(0,1)}[x, y] g 22^{(0,1)}[x, y]+g 22^{(1,0)}[x, y]^{2}\right)\right. \\
& \quad+g 22[x, y]\left(g 11^{(0,1)}[x, y]^{2}+g 11^{(1,0)}[x, y] g 22^{(1,0)}[x, y]\right. \\
& \left.\left.\quad-2 g 11[x, y]\left(g 11^{(0,2)}[x, y]+g 22^{(2,0)}[x, y]\right)\right)\right)^{2} \\
& /\left(4 g 11[x, y]^{4} g 22[x, y]^{4}\right)
\end{aligned}
$$

for the simpler case of a diagonal metric.

We now consider the case of $4 \mathrm{D}$ objects. Let us assume that the metric is diagonal and that the first two elements are equal, that is, the metric is of the form $\operatorname{diag}(g 11[x, y, z]$, $g 11[x, y, z], g 33[x, y, z])$.

With this assumption, invariant (29) can be written as

$$
\begin{aligned}
(2 g 33 & {[x, y, z]^{2}\left(g 11^{(0,1,0)}[x, y, z]^{2}+g 11^{(1,0,0)}[x, y, z]^{2}\right) } \\
& -g 11[x, y, z] g 33[x, y, z]\left(-g 11^{(0,0,1)}[x, y, z]^{2}\right. \\
& \left.+2 g 33[x, y, z]\left(g 11^{(0,2,0)}[x, y, z]+g 11^{(2,0,0)}[x, y, z]\right)\right) \\
& +g 11[x, y, z]^{2}\left(2 g 11^{(0,0,1)}[x, y, z] g 33^{(0,0,1)}[x, y, z]\right. \\
& +g 33^{(0,1,0)}[x, y, z]^{2}+g 33^{(1,0,0)}[x, y, z]^{2} \\
& -2 g 33[x, y, z]\left(2 g 11^{(0,0,2)}[x, y, z]+g 33^{(0,2,0)}[x, y, z]\right. \\
& \left.\left.\left.+g 33^{(2,0,0)}[x, y, z]\right)\right)\right)^{2} \\
/( & \left.4 g 11[x, y, z]^{6} g 33[x, y, z]^{4}\right) .
\end{aligned}
$$

We need three coordinates to describe a 4D object, since the latter is a volumetric image. If all the diagonal elements are equal, that is to say, if the metric is of the form $\operatorname{diag}(g 11[x, y, z], g 11[x, y, z], g 11[x, y, z])$, one obtains

$$
\begin{aligned}
& \left(3 g 11^{(0,0,1)}[x, y, z]^{2}-4 g 11[x, y, z] g 11^{(0,0,2)}[x, y, z]\right. \\
& \quad+3 g 11^{(0,1,0)}[x, y, z]^{2}-4 g 11[x, y, z] g 11^{(0,2,0)}[x, y, z] \\
& \left.\quad+3 g 11^{(1,0,0)}[x, y, z]^{2}-4 g 11[x, y, z] g 11^{(2,0,0)}[x, y, z]\right)^{2} \\
& /\left(4 g 11[x, y, z]^{6}\right)
\end{aligned}
$$

which is of course a much simpler expression. The level of complexity of the expression is not only related to the components of the metric tensor (and consequently the source tensor) but also to the level of symmetry of the later.

Finally, let us assume a traceless metric (i.e., all the diagonal elements are equal to zero) without any other restriction on the other elements. Then, invariant (29) is given by the following complex expression:

$$
\begin{aligned}
& \left(2 g 3 1 [ x , y , z ] g 3 2 [ x , y , z ] \left(g 32[x, y, z] g 21^{(1,0,0)}[x, y, z]\right.\right. \\
& \times\left(g 21^{(0,0,1)}[x, y, z]+g 31^{(0,1,0)}[x, y, z]\right. \\
& \left.-g 32^{(1,0,0)}[x, y, z]\right)+g 31[x, y, z] g 21^{(0,1,0)}[x, y, z] \\
& \left.\times\left(g 21^{(0,0,1)}[x, y, z]-g 31^{(0,1,0)}[x, y, z]+g 32^{(1,0,0)}[x, y, z]\right)\right) \\
& +2 g 21[x, y, z]^{2}\left(g 31[x, y, z] g 32^{(0,0,1)}[x, y, z]\right. \\
& \times\left(-g 21^{(0,0,1)}[x, y, z]+g 31^{(0,1,0)}[x, y, z]\right. \\
& \left.+g 32^{(1,0,0)}[x, y, z]\right)+g 32[x, y, z]\left(-g 21^{(0,0,1)}[x, y, z]\right. \\
& \times g 31^{(0,0,1)}[x, y, z]+g 31^{(0,0,1)}[x, y, z]\left(g 31^{(0,1,0)}[x, y, z]\right. \\
& \left.+g 32^{(1,0,0)}[x, y, z]\right)+2 g 31[x, y, z]\left(g 21^{(0,0,2)}[x, y, z]\right. \\
& \left.\left.\left.-g 31^{(0,1,1)}[x, y, z]-g 32^{(1,0,1)}[x, y, z]\right)\right)\right)+g 21[x, y, z] \\
& \times\left(2 g 3 2 [ x , y , z ] ^ { 2 } g 3 1 ^ { ( 1 , 0 , 0 ) } [ x , y , z ] \left(g 21^{(0,0,1)}[x, y, z]\right.\right. \\
& \left.+g 31^{(0,1,0)}[x, y, z]-g 32^{(1,0,0)}[x, y, z]\right) 2 g 31[x, y, z]^{2} \\
& +\left(g 21^{(0,0,1)}[x, y, z] g 32^{(0,1,0)}[x, y, z]-g 31^{(0,1,0)}[x, y, z]\right. \\
& \times g 32^{(0,1,0)}[x, y, z]-2 g 32[x, y, z] g 21^{(0,1,1)}[x, y, z] \\
& +2 g 32[x, y, z] g 31^{(0,2,0)}[x, y, z]+g 32^{(0,1,0)}[x, y, z] \\
& \left.\times g 32^{(1,0,0)}[x, y, z]-2 g 32[x, y, z] \times g 32^{(1,1,0)}[x, y, z]\right) \\
& +g 31[x, y, z] g 32[x, y, z]\left(g 21^{(0,0,1)}[x, y, z]^{2}\right. \\
& +g 31^{(0,1,0)}[x, y, z]^{2}-2 g 31^{(0,1,0)}[x, y, z] g 32^{(1,0,0)}[x, y, z] \\
& +g 32^{(1,0,0)}[x, y, z]^{2}-2 g 21^{(0,0,1)}[x, y, z] \\
& \times\left(g 31^{(0,1,0)}[x, y, z]+g 32^{(1,0,0)}[x, y, z]\right)-4 g 32[x, y, z] \\
& \times g 21^{(1,0,1)}[x, y, z]-4 g 32[x, y, z] g 31^{(1,1,0)}[x, y, z] \\
& \left.\left.\left.+4 g 32[x, y, z] g 32^{(2,0,0)}[x, y, z]\right)\right)\right)^{2} \\
& /\left(16 g 21[x, y, z]^{4} g 31[x, y, z]^{4} g 32[x, y, z]^{4}\right),
\end{aligned}
$$

where $(2,1,0)$ is a partial with respect to $z, y$, and $x$. A similar notation applies to the other derivatives. 
Consequently, we have obtained exact expressions for invariant (29) for 3D objects for a general and a diagonal metric. Moreover, for 4D objects, we obtained exact expressions for invariant (29), for a diagonal metric for which all the elements are equal, for a diagonal metric for which two elements are equal as well as for a traceless metric.

To conclude this section, we would like to present some numerical experimental results for 3D objects. In the following, all the objects are described with invariant (29) and with representation (32), that is, the index or descriptor is a histogram of the square of the Ricci tensor. All our calculations were performed with the Viewpoint Datalab libraries and collections. This repository consists, in our edition, of 12.150 (twelve thousand) objects of a variety of objects such as cars, planes, human bodies, heads, trees, just to mention a few.

With these examples, we illustrate that our method can retrieve an object that has been submitted to a general coordinate transformation or GCT and that such invariance does not deteriorate the discrimination level. That is one of the reasons why we consider such a large database. In addition, we show that the proposed method can be utilised to retrieve similar objects, that is, the method is not limited to identical objects submitted to GCT.

The numerical implementation of the calculation will be the subject of another publication. In essence, we employ stochastic or Monte Carlo methods [11] in order to drastically reduce the amount of calculation. The Monte Carlo sampling does not provide an exact result, but an approximation, which, as far as the experimental results are involved, is sufficient for the size (12.150 items) and composition of our database. As a first example, let us consider Figure 2.

Figure 2 represents a character which was animated with various facial expressions. Such a variation of the facial expression is equivalent to a GCT. We applied our method to this character and retrieved all his facial expression without any inlayer (precision: $100 \%$; recall: $100 \%$ ). Such a result indicates the efficiency of the method both in terms of invariance under a GCT as well as in terms of discrimination. In our second example, we consider Figure 3, which illustrates a query for a car.

We managed to retrieve approximately $90 \%$ of the cars present in the database without any inlayer (precision: 100\%; recall: $90 \%$ ). This example shows that the proposed method can be applied, not only to identical objects submitted to a GCT, but also to similar or related objects. Comparable results were obtained for planes and are illustrated in Figure 4. Most of the planes were retrieved without any inlayer, despite the fact that the resolution of the reference model was very low (precision: 100\%; recall: 80\%).

In our final example, we consider Figure 5 which illustrates a query for an animated body. Here, the woman's arms are in two different positions. Such an animation corresponds to a $\mathrm{GCT}$.

Again, we managed to retrieve both postures without any inlayer. The next retrieved items (up to rank 250) were all human bodies without any inlayer (precision: 100\%; recall: $100 \%)$. That shows, once more, that the method is invariant under general coordinate transformation and suitable to

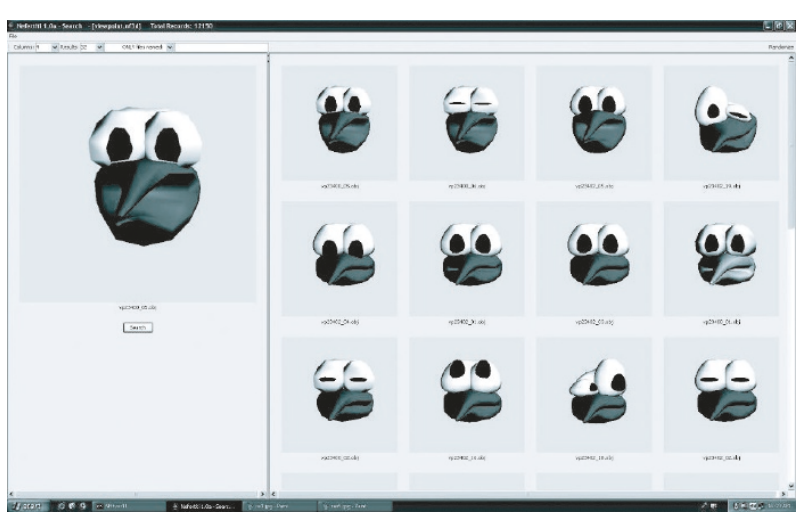

FIGURE 2: Retrieval of an animated 3D character: the reference object appears on the left side while the outcome of the query appears on the right side. Each result is characterised by a different facial expression, that is, a GCT. In the present query, all the facial expressions of the character were retrieved without any inlayer from a database containing 12.150 objects.

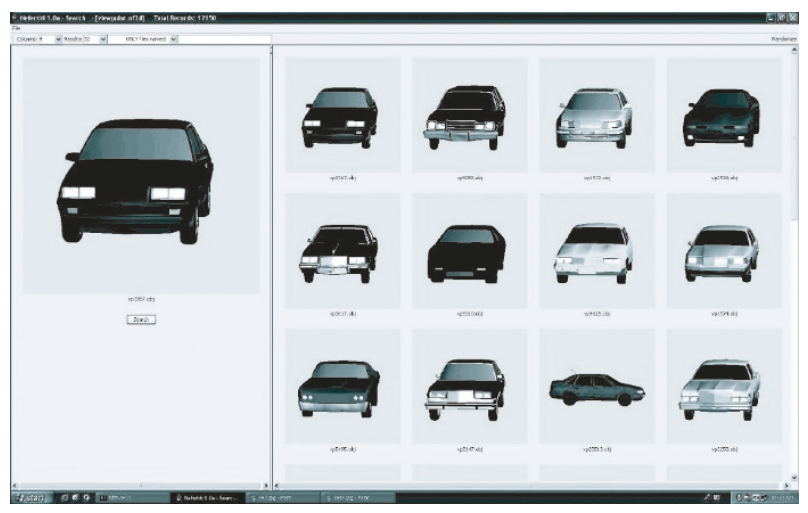

FIGURE 3: Retrieval of cars. Most of the cars (approximately 90\%) were retrieved without inlayers from the 12.150 objects database. Only the first results are displayed.

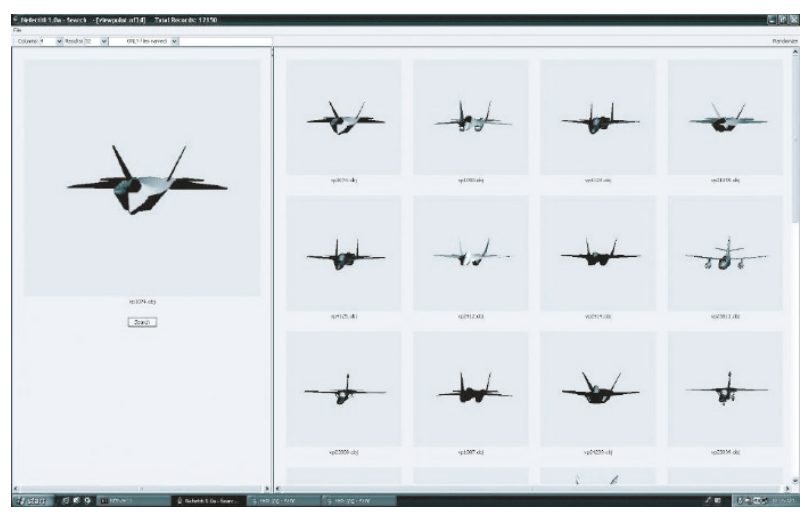

Figure 4: Retrieval of planes. We retrieved most of the planes (approximately $80 \%$ ) without inlayer despite the fact that the reference model had a very low resolution. Only the first results are shown. 


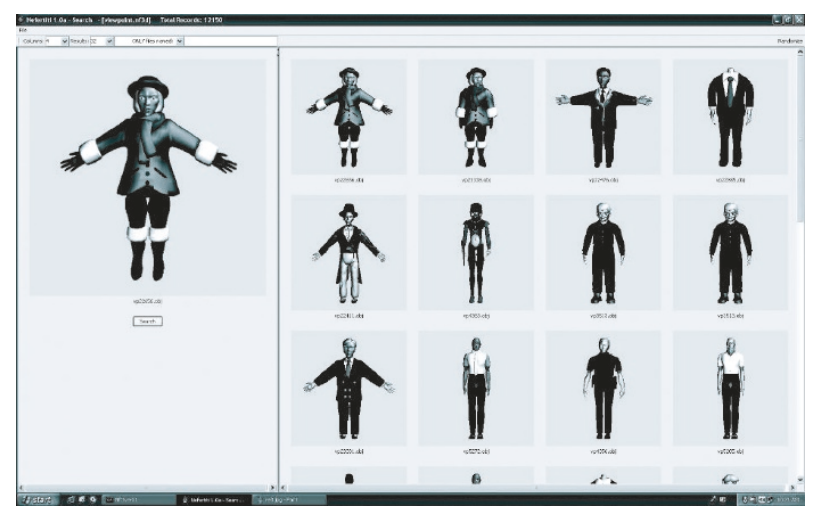

FIgURE 5: Retrieval of an animated 3D character. We retrieved all (i.e., 2) the postures associated with the mannequin and most of the human bodies from the 12.150 objects database. Only the first results are shown.

retrieve similar object while maintaining an adequate discrimination level.

The above-mentioned examples, as many others that are not shown in the present paper, indicate that the proposed method is efficient to retrieve $3 \mathrm{D}$ objects submitted to a GCT as well as similar objects from a large database. The fact that the database is large (12.150 objects) shows, at least from a statistical point of view, that the invariance under GCT does not compromise the level of discrimination of the algorithm.

\section{CONCLUSIONS}

In this paper, we have associated a curved space to an arbitrary object and have described this space with quantities that are invariant under general coordinate transformations. From those quantities we have built two representations: one based on the statistical distribution of the invariants and the other based on their topological distribution. Both representations are invariant under GCT. Promising experimental results were provided both analytically and numerically for a database of $12.1503 \mathrm{D}$ objects.

To the best of our knowledge, there are no approaches that propose such a general and formal framework for GCT invariant representations of object. The next step will be to implement the proposed method, meaning solving exactly (27). This will be achieved through a foliation algorithm which will be implemented on a grid computer. In addition, I propose to study various approximations to (27) that would be precise enough for indexing and retrieval and that would facilitate and speed up the calculations.

\section{REFERENCES}

[1] N. Iyer, S. Jayanti, K. Lou, Y. Kalyanaraman, and K. Ramani, "Three-dimensional shape searching: state-of-the-art review and future trends," Computer Aided Design, vol. 37, no. 5, pp. 509-530, 2005.

[2] J. W. H. Tangelder and R. C. Veltkamp, "A survey of content based 3D shape retrieval methods," in Proceedings of IEEE
International Conference on Shape Modeling and Applications (SMI '04), pp. 145-156, Genova, Italy, June 2004.

[3] A. Theetten, J.-P. Vandeborre, and M. Daoudi, "Determining characteristic views of a 3D object by visual hulls and Hausdorff distance," in Proceedings of 5th International Conference on 3-D Digital Imaging and Modeling, pp. 439-446, Los Alamitos, Calif, USA, 2005.

[4] D. V. Vranic and D. Saupe, "Description of 3D-shape using a complex function on the sphere," in Proceedings of IEEE International Conference on Multimedia and Expo (ICME '02), vol. 1, pp. 177-180, Lausanne, Switzerland, August 2002.

[5] M. Göckeler and T. Schücker, Differential Geometry, Gauge Theories, and Gravity, Cambridge University Press, New York, NY, USA, 1989.

[6] C. Rovelli, Quantum Gravity, Cambridge University Press, New York, NY, USA, 2004.

[7] C. Kiefer, Quantum Gravity, Oxford University Press, New York, NY, USA, 2004.

[8] D. Lovelock and H. Rund, Tensors, Differential Forms and Variational Principles, Dover, New York, NY, USA, 1989.

[9] C. Bona and C. Palenzuela-Luque, Elements of Numerical Relativity, Springer, New York, NY, USA, 2005.

[10] S. Wolfram, The Mathematica Book, Wolfram Media, Champaign, Ill, USA, 5th edition, 2003.

[11] C. P. Robert and G. Casella, Monte Carlo Statistical Methods, Springer, New York, NY, USA, 1999.

Eric Paquet is a Senior Research Officer at the Visual Information Technology (VIT) Group of the National Research Council of Canada (NRC). He received his Ph.D. degree in computer vision from Laval University and the National Research Council in 1994. After finishing his Ph.D., he worked on optical information processing in Spain, on laser microscopy at the Technion-Israel Institute of Technology, and on 3D hand-

held scanners in England. He is pursuing research on contentbased management of multimedia information and applied visualisation at the National Research Council of Canada. His current research interests include content-based description of multimedia and multidimensional objects, anthrometric databases, and cultural heritage applications. He is the author of numerous publications, Member of MPEG, WEAR, CAESAR, ISPRS, SCC, CODATA, and Member on the programme committee of several international conferences, and has received many international awards. 